\title{
Investigation of bifurcation properties of a model of myotonia caused by incomplete inactivation of sodium channels in skeletal muscle fibers
}

\author{
Kamonwan Kocharoen ${ }^{1}$ and Jonathan Bell*2
}

\author{
Address: ${ }^{1}$ Department of Mathematics, Mahidol University, Bangkok, Thailand, 10400 and ${ }^{2}$ Department of Mathematics and Statistics, University \\ of Maryland Baltimore County, Baltimore, Maryland, 21250 USA \\ Email: Jonathan Bell* - jbell@math.umbc.edu \\ * Corresponding author
}

from Sixteenth Annual Computational Neuroscience Meeting: CNS*2007

Toronto, Canada. $7-12$ July 2007

Published: 6 July 2007

BMC Neuroscience 2007, 8(Suppl 2):PI25 doi:10.1 186/I47I-2202-8-S2-PI25

(c) 2007 Kocharoen and Bell; licensee BioMed Central Ltd.

Muscle fibers from people suffering a myotonia condition generate trains of action potentials when extracellular potassium to the fiber is elevated. In milder cases of myotonia this leads to muscle stiffness, while in more severe cases the muscle can experience partial paralysis. The pathological features arise mainly from mutations of the sodium channel that causes a partial loss of inactivation. These features can be simulated in rat skeletal muscle by applying a toxin (anemonia toxin ATX II) in vitro to the muscle. Cannon, et al [1] formulated a two-compartment model representing the sarcolemma and t-tubule system of a skeletal muscle fiber, employing Hodgkin-Huxley type dynamics, with parameter values of mammalian muscle at room temperature, and compared its behavior to ATX II-affected rat muscle. A parameter, $\mathrm{f}$, representing the fraction of sodium channels in the sarcolemma with defective inactivation dynamics could be adjusted to simulate normal, myotonic, and paralysis features in the muscle. To better understand features of their 9 equation model, we first reduced the model to considering just the t-tubule potential and potassium activation dynamics, depending on the parameter $f$ and extracellular potassium concentration Kt. Over the physiological range in $\mathrm{f}$, Kt space we obtain regions of multiple equilibrium states, and their bifurcation properties, mostly saddle-node and Hopf bifurcations, and identify the onset of large limit cycle behavior representing post-stimulus repetitive discharging. Because coldness can bring on the episodic symptoms, we then examine the sensitivity of the model to temperature, as well as with other parameters. The reduced model is too simple to produce paralytic effects, so we consider other interactions of Cannon's two-compartment model formulation. This study is preliminary in that more dynamics is slowly being incorporated into the model study, including spatial effects.

\section{References}

I. Cannon SC, Brown RH, Corey DP: Theoretical reconstruction of myotonia and paralysis caused by incomplete inactivation of sodium channels. Biophys J 1993, 65:270-288. 